\title{
EFICIÊNCIA DEA COMO MEDIDA DE DESEMPENHO DE UNIDADES POLICIAIS
}

\section{DEA EFFICIENCY SCORE AS PERFORMANCE INDICATOR OF POLICE UNITS}

\author{
João Carlos Correia Baptista Soares de Mello \\ Professor Adjunto \\ Universidade Federal Fluminense \\ Departamento de Engenharia de Produção \\ Rua Passo da Pátria 156, São Domingos, 24210-240, Niterói, RJ \\ Tel.: (0xx21) 26295378 \\ jcsmello@producao.uff.br
}

\section{Eliane Gonçalves Gomes}

Pesquisador

Embrapa

Parque Estação Biológica, W3 Norte final, Asa Norte, 70770-901, Brasília, DF eliane.gomes@embrapa.br

\author{
Altair Souza de Assis \\ Professor Titular \\ Universidade Federal Fluminense \\ Departamento de Matemática Aplicada
}

Rua São Paulo s/n, Valonguinho, 24001-970, Niterói, RJ

gmaasda@vm.uff.br 


\title{
David Pereira Morais
}

\author{
Pesquisador \\ Universidade Cândido Mendes \\ Diretoria de Projetos Especiais
}

Rua da Assembléia 10 - sala 4208, Centro, 20011-901, Rio de Janeiro, RJ

dmorais@candidomendes.edu.br

\section{RESUMO}

O governo do Estado do Rio de Janeiro instituiu uma premiação para as unidades policiais que mais se destacaram em cada mês. O Estado foi dividido em Áreas Integradas de Segurança Pública (AISPs), que compreendem um batalhão de polícia militar e delegacia(s) de polícia civil. As AISPs são agrupadas segundo a região a que pertençam (Capital, Metropolitana e Interior). O prêmio é atribuído a AISP, em cada região, que apresentou os melhores indicadores de produção nas ações de combate ao crime. Ao considerar apenas a produção, esta premiação não considera os meios utilizados para realizar as ações. É necessário comparar as ações de combate ao crime (outputs) com os recursos e com o nível de criminalidade da região. A Análise de Envoltória de Dados provê um meio eficaz de fazer tal comparação, pois permite ordenar as AISPs segundo um índice de eficiência e indicar aquelas que devam servir de exemplo para as ineficientes. O modelo DEA BCC é usado neste artigo para ordenar apenas os batalhões, considerando-se que um batalhão em meses diferentes é uma unidade diferente.

Palavras-chave: Serviços públicos - Segurança pública - Análise de Envoltória de Dados.

\section{ABSTRACT}

The Rio de Janeiro State government uses to monthly evaluate police units. For that mater the state was divided into Public Security Integrated Areas (PSIAs), which comprise both military and civil units. PSIAs are aggregated by the areas they belong to (Capital, Metropolitan and Interior). A prize is given to the PSIA, in each area, which had the best evaluation in crime combating. Considering only production indicators, this prize doesn't take into account the 
resources employed in these actions. It is important to compare the crime combating actions (outputs) with the resources available and the criminality level in the PSIAs. Data Envelopment Analysis (DEA) is an appropriate approach to perform these comparisons, as it ranks the PSIAs by an efficiency score and also points out the benchmarks. BCC DEA model is used in this paper to rank only the police battalion, considering that one battalion in a different month is a different evaluation unit.

Key-words: Public services - Public security - Data Envelopment Analysis.

\section{INTRODUÇÃO}

O Estado do Rio de Janeiro vem há vários anos enfrentando problemas relativos à falta de segurança, em especial em seus grandes centros urbanos. Uma das medidas tomadas pelo governo empossado em 1999 para enfrentar essa situação foi a instituição de uma premiação para as unidades policiais que mais se destacaram em cada mês.

A possibilidade de estabelecer uma premiação para as áreas de maior "eficiência policial" foi considerada com base na proposta de dividir o Estado em Áreas Integradas de Segurança Pública (AISPs). As AISPs foram concebidas como unidades descentralizadas de planejamento, controle, supervisão, avaliação e monitoramento corretivo das atividades de segurança pública, e também como referências para a indução ao bom desempenho conjunto dos policiais (por meio de estímulos, gratificações e premiações baseadas no desempenho da área como um todo), assim como para a punição de condutas violentas, corruptas e arbitrárias. Trata-se de 36 circunscrições territoriais sob responsabilidade compartilhada pelo comandante do batalhão e pelo(s) delegado(s) titular(es) da(s) delegacia(s) situada(s) no mesmo território, e agregam, além disso, outras agências prestadoras de serviços públicos atuantes na respectiva área.

As AISPs são agrupadas segundo a região a que pertençam, a saber, Capital, Metropolitana e Interior. Em cada região o prêmio é atribuído à AISP que apresentou os melhores indicadores de produção nas ações de combate ao crime. Essa produção considera, entre outros, prisões efetuadas e apreensão de drogas.

No Estado do Rio de Janeiro estão constituídas 17 AISPs na Capital (município do Rio de Janeiro), 7 na região Metropolitana (municípios de São Gonçalo, Niterói, Maricá, Duque de Caxias, Nova Iguaçu, Belford Roxo, Nilópolis, São João de Meriti, Seropédica, Itaguaí, Paracambi, Queimados, Japeri, Magé, Guapimirim) e 12 AISPs no Interior (75 municípios). 
Embora oficialmente os resultados das ações em cada AISP sejam apresentados como produtividade, eles não levam em conta os meios disponíveis para sua obtenção e são, portanto, uma medida de produção.

Como o objetivo é comparar as AISPs, a abordagem por Análise de Envoltória de Dados (Data Envelopment Analysis - DEA) provê um meio eficaz de fazer tal comparação, pois permite ordená-las segundo um índice de eficiência multidimensional. Permite ainda indicar as AISPs que devem servir de exemplo (benchmarks) para as ineficientes. As variáveis usadas na modelagem estão ligadas à quantidade de delitos praticados, à produção policial (única variável considerada atualmente para a atribuição do prêmio) e ao efetivo policial.

Assim, este artigo expande os resultados de Soares de Mello et al. (2004) e propõe o uso do modelo DEA BCC para avaliar os batalhões (já que os dados das delegacias não estavam disponíveis). Considera-se que um batalhão em meses diferentes é uma unidade diferente.

\section{ANÁLISE DE ENVOLTÓRIA DE DADOS}

A Análise de Envoltória de Dados surgiu formalmente em 1978 com o trabalho de Charnes et al. (1978), com o objetivo de medir a eficiência de unidades tomadoras de decisão, designadas por DMUs (Decision Making Units), na presença de múltiplos fatores de produção (inputs) e múltiplos produtos (outputs).

Há dois modelos DEA clássicos: CCR (de Charnes, Cooper e Rhodes) e BCC (de Banker, Charnes e Cooper). O modelo CCR (também conhecido por CRS ou Constant Returns to Scale), trabalha com retornos constantes de escala (CHARNES et al., 1978). Em sua formulação matemática considera-se que cada DMU $k, k=1 \ldots s$, é uma unidade de produção que utiliza $n$ inputs $x_{i k}, i=1 \ldots n$, para produzir $m$ outputs $y_{j k}, j=1 \ldots m$. Esse modelo maximiza o quociente entre a combinação linear dos outputs e a combinação linear dos inputs, com a restrição de que para qualquer DMU esse quociente não pode ser maior que 1.

Mediante alguns artifícios matemáticos, este modelo pode ser linearizado, transformando-se em um Problema de Programação Linear (PPL) apresentado em (1), onde $h_{o}$ é a eficiência da DMU $o$ em análise; $x_{i o}$ e $y_{j o}$ são os inputs e outputs da DMU $o$; $v_{i}$ e $u_{j}$ são os pesos calculados pelo modelo para inputs e outputs. 
$\max h_{o}=\sum_{j=1}^{m} u_{j} y_{j o}$

sujeito a

$\sum_{i=1}^{n} v_{i} x_{i o}=1$

$\sum_{j=1}^{m} u_{j} y_{j k}-\sum_{i=1}^{n} v_{i} x_{i k} \leq 0, \quad k=1, \ldots, s$

$u_{j}, v_{i} \geq 0 \quad \forall x, y$

O modelo BCC, também chamado de VRS (Variable Returns to Scale) considera situações de eficiência de produção com variação de escala e não assume proporcionalidade entre inputs e outputs. Apresenta-se em (2) a formulação do deste modelo (BANKER et al., 1984), com orientação à expansão de produtos. Em (2), $h_{o}$ é a eficiência da DMU $o$ em análise; $x_{i k}$ representa o input $i$ da $\mathrm{DMU}_{k}, y_{j k}$ representa o output $j$ da $\mathrm{DMU}_{\mathrm{k}} ; v_{i}$ é o peso atribuído ao input $i, u_{j}$ é o peso atribuído ao output $j ; v_{*}$ é um fator de escala.

$$
\begin{aligned}
& \text { Min } E f f_{o}=\sum_{i=1}^{n} v_{i} x_{i o}-v_{*} \\
& \text { sujeito a } \\
& \sum_{j=1}^{m} u_{j} y_{j o}=1 \\
& -\sum_{i=1}^{n} v_{i} x_{i k}+\sum_{j=1}^{m} u_{j} y_{j k}-v_{*} \leq 0, \quad \forall k \\
& u_{j}, v_{i} \geq 0, \forall j, i \\
& v_{*} \in \mathfrak{R}
\end{aligned}
$$

A Figura 1 mostra as fronteiras DEA BCC e CCR para um modelo DEA bidimensional (1 input e 1 output). As DMUs A, B e C são BCC eficientes; a DMU B é CCR eficiente. As DMUs D e E são ineficientes nos dois modelos. A eficiência CCR e BCC da DMU E, para orientação a outputs, é dada, respectivamente, por $\frac{\overline{\mathrm{E}^{\prime \prime} \mathrm{E}}}{\overline{\mathrm{E}^{\prime \prime} \mathrm{E}^{\prime \prime \prime}}}$ e $\frac{\overline{\mathrm{E}^{\prime \prime} \mathrm{E}}}{\overline{\mathrm{E}^{\prime \prime} \mathrm{E}^{\prime}}}$. 


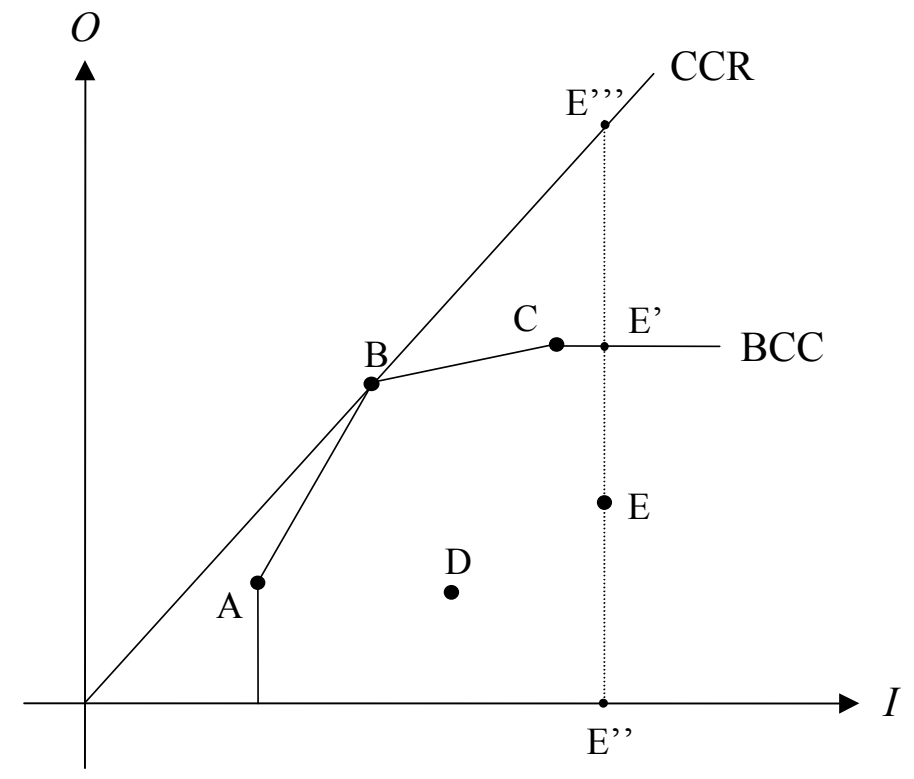

Figura 1: Fronteiras DEA BCC e CCR para o caso bidimensional.

Além de identificar as DMUs eficientes, os modelos DEA permitem medir e localizar a ineficiência e estimar uma função de produção linear por partes, que fornece o benchmark para as DMUs ineficientes. Esse benchmark é determinado pela projeção das DMUs ineficientes na fronteira de eficiência. A forma como é feita esta projeção determina orientação do modelo: orientação a inputs (quando se deseja minimizar os inputs, mantendo os valores dos outputs constantes) e orientação a outputs (quando se deseja maximizar os resultados sem diminuir os recursos).

\section{ESTUDO DE CASO: EFICIÊNCIA DA SEGURANÇA PÚBLICA}

\subsection{Modelagem}

A modelagem em DEA exige a definição das DMUs, das variáveis de avaliação (inputs e outputs) e do modelo DEA que será usado.

As DMUs são os batalhões de polícia militar correspondentes a cada AISP. Para garantir a desejável homogeneidade entre as unidades em avaliação, elas serão agrupadas em três conjuntos correspondentes às regiões de atuação instituídas oficialmente. Embora alguns municípios do Interior tenham atualmente características socioeconômicas semelhantes às de 
região Metropolitana, optou-se por manter a divisão oficial pela insuficiência de dados que permitam uma divisão mais adequada.

Uma vez agregadas as AISPs em regiões, supõe-se que elas tenham escalas semelhantes e, portanto, será usado o modelo DEA CCR. No entanto, é interessante comparar todas as AISPs do Estado, classificadas segundo um mesmo índice. Como nessa situação existem diferenças de escalas apreciáveis, é conveniente o uso do modelo DEA BCC. Ambos os modelos são orientados a outputs.

Os dados utilizados foram obtidos no Diário Oficial do Estado do Rio de Janeiro e referem-se aos meses de janeiro e fevereiro de 2002. Para permitir o acompanhamento da evolução temporal das unidades em avaliação, um mesmo batalhão em meses diferentes é considerado como uma DMU diferente, segundo um procedimento análogo ao adotado por Soares de Mello et al. (2003).

As variáveis usadas referem-se à quantidade de delitos praticados, à produção policial e ao efetivo policial. A Tabela 1 apresenta algumas estatísticas descritivas para as variáveis usadas.

Tabela 1: Estatísticas descritivas para as variáveis usadas na modelagem.

\begin{tabular}{|c|c|c|c|}
\cline { 2 - 4 } \multicolumn{1}{c|}{} & Delitos praticados & Produção Policial & Efetivo policial \\
\hline Média & 169 & $1.441,1$ & 80.401 \\
Mínimo & 4 & 12,1 & 25.920 \\
$1^{\circ}$ Quartil & 67 & 123,8 & 54.293 \\
$2^{\circ}$ Quartil & 106 & 398,6 & 70.869 \\
$3^{\circ}$ Quartil & 231 & $1.178,5$ & 100.871 \\
Máximo & 825 & $30.939,8$ & 212.900 \\
Desvio padrão & 161 & $3.939,0$ & 37.476 \\
\hline
\end{tabular}

A variável efetivo policial considera apenas os policiais envolvidos em atividade fim e é, claramente, um input. A quantidade de delitos praticados enfrenta a dificuldade da agregação de delitos de natureza diferente. Este problema multicritério não será aqui abordado e a agregação tomou como base o índice oficial usado para a premiação das AISPs. Essa quantidade total de delitos é um tipo de output conhecido na literatura DEA como output indesejável (SCHEEL, 2001). 
Esta variável necessita de discussão adicional. Se fosse levado em conta o registro de delitos, em vez da ocorrência de delito, ela seria um output, já que representaria a produção de uma entidade burocrática. No entanto, para a segurança pública, o mais importante é que não ocorram delitos e, por isso, a variável será considerada um output indesejável.

Em uma primeira abordagem, poder-se-ia pensar que ela é uma variável ambiental, já que descreve o que acontece na região em que a unidade policial está instalada, sem influência da dita unidade. Ao ser válido este ponto de vista, a quantidade de delitos praticados seria claramente um input não controlável, e deveria ser usado o modelo específico para esta situação (BANKER, MOREY, 1986; SYRJÄNEN, 2004). No entanto, este ponto de vista só é válido para uma unidade policial que tenha sido recentemente instalada na região, onde lida com a criminalidade já existente. Ou seja, é uma abordagem válida apenas em regime transiente. Já em regime permanente, isto é, quando a unidade policial está instalada na região há um tempo razoável, a existência (ou ausência) de criminalidade é, em grande parte, resultado direto da sua atuação. Assim, a quantidade de delitos é uma medida (talvez a mais importante) da falta de eficácia policial. Portanto, é, claramente, um output indesejável e, pelo menos parcialmente, controlável.

Como tal, há duas maneiras de tratá-lo: como input, que pode ser usado tanto em modelos DEA BCC quanto em CCR; ou como um output, cujo valor é o seu inverso. Esta abordagem só pode ser usada em modelos DEA CCR. Neste artigo, como serão usados os dois tipos de modelos, os delitos são considerados inputs, em abordagem semelhante à usada por Sun (2002).

As ações realizadas no combate à criminalidade são o output, agregado segundo os pesos usados pela Secretaria Estadual de Segurança Pública.

Os modelos usados buscam retratar a capacidade de cada AISP em combater a criminalidade existente com os recursos de que dispõe. Deve ser ressaltado que uma AISP que atue em uma área de baixa criminalidade não precisa realizar muitas ações para ser considerada eficiente. Isto está de acordo com o que a sociedade espera do serviço policial, ou seja, manutenção de baixos índices de criminalidade e não a realização de ações emergenciais.

\subsection{Resultados}

A Tabela 2 apresenta os resultados das AISPs de melhor eficiência para as três regiões, no modelo DEA CCR, e suas estatísticas descritivas. As eficiências foram obtidas 
com o programa SIAD - Sistema Integrado de Apoio à Decisão (ANGULO MEZA et al., 2005).

Tabela 2: Estatísticas descritivas dos resultados de eficiência das AISPs, segundo as regiões.

\begin{tabular}{|c|ccc|ccc|ccc|}
\cline { 2 - 10 } \multicolumn{1}{c|}{} & \multicolumn{3}{c|}{ Interior } & \multicolumn{3}{c|}{ Capital } & \multicolumn{3}{c|}{ Metropolitana } \\
\cline { 2 - 10 } \multicolumn{1}{c|}{} & Global & Jan. & Fev. & Global & Jan. & Fev. & Global & Jan. & Fev. \\
\hline N. $^{\text {o }}$ DMUs eficientes & 2 & 0 & 2 & 1 & 1 & 0 & 1 & 1 & 0 \\
\hline Média & 0,1827 & 0,1083 & 0,2570 & 0,0725 & 0,0911 & 0,0539 & 0,2200 & 0,2419 & 0,1980 \\
Mínimo & 0,0217 & 0,0233 & 0,0217 & 0,0008 & 0,0013 & 0,0008 & 0,0217 & 0,0345 & 0,0217 \\
$1^{\circ}$ Quartil & 0,0376 & 0,0404 & 0,0376 & 0,0056 & 0,0072 & 0,0044 & 0,0366 & 0,0528 & 0,0321 \\
$2^{\circ}$ Quartil & 0,0723 & 0,0746 & 0,0723 & 0,0179 & 0,0179 & 0,0179 & 0,1017 & 0,1024 & 0,1010 \\
$3^{\circ}$ Quartil & 0,1304 & 0,1135 & 0,2430 & 0,0512 & 0,0516 & 0,0500 & 0,2629 & 0,2255 & 0,2461 \\
Máximo & 1,0000 & 0,3428 & 1,0000 & 1,0000 & 1,0000 & 0,2599 & 1,0000 & 1,0000 & 0,7072 \\
Desvio padrão & 0,2762 & 0,1063 & 0,3690 & 0,1753 & 0,2378 & 0,0781 & 0,2924 & 0,3465 & 0,2530 \\
\hline
\end{tabular}

Na Tabela 3 mostram-se as eficiências das AISPs quando avaliadas conjuntamente no modelo DEA BCC.

Tabela 3: Resultados de eficiência para o caso da avaliação conjunta.

\begin{tabular}{|c|c|c|c|c|}
\cline { 2 - 5 } \multicolumn{1}{c|}{} & Global & Interior & Capital & Metropolitana \\
\hline N. ${ }^{\text {o }}$ DMUs eficientes & 5 & 4 & 1 & 0 \\
\hline Média & 0,1399 & 0,0821 & 0,2604 & 0,0739 \\
Mínimo & 0,0011 & 0,0011 & 0,0090 & 0,0050 \\
$1^{\text {o }}$ Quartil & 0,0128 & 0,0067 & 0,0206 & 0,0121 \\
$2^{\text {o }}$ Quartil & 0,0317 & 0,0209 & 0,0523 & 0,0203 \\
$3^{\text {o }}$ Quartil & 0,0986 & 0,0732 & 0,3214 & 0,0623 \\
Máximo & 1,0000 & 1,0000 & 1,0000 & 0,5523 \\
Desvio padrão & 0,2650 & 0,1801 & 0,3687 & 0,1434 \\
\hline
\end{tabular}

A análise dos resultados mostra que a região Metropolitana teve maior índice de eficiência em janeiro, ocorrendo o inverso no Interior. A análise conjunta revela que a maioria das unidades eficientes está localizada no Interior, o que está de acordo com a percepção 
pública de que há maior criminalidade nas grandes cidades, onde é mais difícil conseguir-se uma boa atuação policial.

A análise dos benchmarks é apresentada na Tabela 4 para os quatro modelos usados (3 modelos DEA CCR, um para cada região, e 1 modelo DEA BCC, considerando todas as regiões conjuntamente). Verifica-se que uma AISP da Capital no mês de janeiro e uma AISP do Interior no mês de fevereiro foram eficientes tanto no modelo com regiões separadas quanto no modelo conjunto. Essas AISPs deveriam ser analisadas e, possivelmente, usadas como "espelho" para as demais.

Tabela 4: Valores das variáveis para os benchmarks, segundo o modelo desagregado por região.

\begin{tabular}{|c|ccccc|}
\cline { 2 - 6 } \multicolumn{1}{c|}{} & Região & Mês & Delitos & Produção & Efetivo \\
\hline \multirow{3}{*}{ Modelos com } & Interior & fevereiro & 6 & 475,5 & 89702 \\
separação das regiões & Interior & fevereiro & 63 & 4614,1 & 39600 \\
& Capital & janeiro & 166 & 30939,8 & 65316 \\
& Metropolitana & janeiro & 36 & 2943,8 & 51406 \\
\hline \multirow{3}{*}{ Modelo sem } & Capital & janeiro & 166 & 30939,8 & 65316 \\
separação das regiões & Interior & Janeiro & 37 & 323,9 & 25920 \\
& Interior & Janeiro & 4 & 17,9 & 54312 \\
& Interior & fevereiro & 6 & 475,5 & 89702 \\
& Interior & fevereiro & 6 & 32,5 & 51208 \\
\hline
\end{tabular}

Os resultados do modelo $\mathrm{BCC}$ ainda indicam que das 36 AISPs, operavam em região de retornos crescentes de escala 6 da Capital e 3 da região Metropolitana. Na região da fronteira com retornos decrescentes estavam 10 AISPs da Capital, 12 do Interior e 3 da área Metropolitana, sendo que todas as unidades BCC eficientes estavam nessa região. Ainda, uma AISP da Capital e outra da região Metropolitana operaram com retornos crescentes no mês de janeiro e com retornos decrescentes no mês de fevereiro. Esse modelo não identificou DMUs operando com retornos constantes de escala. 


\section{CONCLUSÕES}

Os modelos usados, embora possam ainda apresentar algumas deficiências, consideram as possibilidades de cada AISP obter resultados e, por isso, mostram-se mais justos que o modelo de premiação oficial. Com efeito, este, ao considerar apenas as ações policiais realizadas, pune as AISPs que atuem em áreas em que conseguem manter baixa criminalidade. Manter baixa criminalidade deve ser considerado o verdadeiro objetivo do serviço policial e, assim, essas AISPs deveriam ser premiadas. Isso acontece nos modelos propostos, em especial no modelo DEA BCC.

Os modelos apresentados são apenas uma primeira abordagem. Deve-se incluir no futuro um estudo sobre a validade de englobar todas as DMUs em um único modelo DEA.

Deve-se ainda tratar algumas inconsistências do modelo DEA BCC (DMUs que atingem eficiência de $100 \%$ ao considerar apenas um único input ou um único output) e considerar que os dados disponíveis não são totalmente precisos. O tratamento destas inconsistências depende de desenvolvimentos teóricos em curso, como o uso da dupla envoltória e de fronteiras difusas (SOARES DE MELLO et al., 2005).

O uso destas técnicas adicionais deverá ainda permitir o desempate entre as DMUs que obtiveram eficiência de $100 \%$. Embora todas elas possam ser consideradas referências para as unidades ineficientes, os múltiplos empates prejudicam análises mais detalhadas.

\section{AGRADECIMENTOS}

Ao CNPq pelo apoio financeiro, processo 301095/2003-5, e a Amaro Cezar Rangel Ferreira (FAETEC) e Newton Cardoso de Oliveira (UCAM) pelas contribuições dadas a este artigo.

\section{REFERÊNCIAS}

ANGULO MEZA, L.; BIONDI NETO, L.; SOARES DE MELLO, J.C.C.B.; GOMES, E.G.; COELHO, P.H.G. ISYDS - Integrated System for Decision Support (SIAD - Sistema Integrado de Apoio a Decisão): A Software Package for Data Envelopment Analysis Model. Pesquisa Operacional, v. 25, n. 3, 2005. (no prelo) 
BANKER, R.D.; CHARNES, A.; COOPER, W.W. Some models for estimating technical scale inefficiencies in Data Envelopment Analysis. Management Science, v. 30, n. 9, p. 10781092, 1984.

BANKER, R.D.; MOREY, R. Efficiency analysis for exogenously fixed inputs and outputs. Operations Research, v. 34, n. 4, p. 513-521, 1986.

CHARNES, A.; COOPER, W.W.; RHODES, E. Measuring the efficiency of decisionmaking units. European Journal of Operational Research, v. 2, p. 429-444, 1978.

SCHEEL, H. Undesirable outputs in efficiency evaluations. European Journal of Operational Research, v. 132, p. 400-410, 2001.

SOARES DE MELLO, J.C.C.B.; ANGULO MEZA, L.; GOMES, E.G.; SERAPIÃO, B.P.; LINS, M.P.E.. Análise de Envoltória de Dados no estudo da eficiência e dos benchmarks para companhias aéreas brasileiras. Pesquisa Operacional, v. 23, n. 2, p. 325-345, 2003.

SOARES DE MELLO, J.C.C.B.; GOMES, E.G.; ANGULO MEZA, L.; BIONDI NETO, L.; SANT'ANNA, A.P. Fronteiras DEA difusas. Investigação Operacional, v. 25, 2005. (no prelo)

SOARES DE MELLO, J.C.C.B.; GOMES, E.G.; ASSIS, A.S.; MORAIS, D.P. Avaliação de eficiência em segurança pública com Análise Envoltória de Dados. In: VII Simpósio de Pesquisa Operacional e Logística da Marinha, 2004, Niterói. Anais... Rio de Janeiro: Centro de Análise de Sistemas Navais, 2004. 1 CD-ROM.

SUN, S. Measuring the relative efficiency of police precints using data envelopment analysis. Socio-Economic Planning Sciences, v. 36, p. 51-71, 2002.

SYRJÄNEN, M.J. Non-discretionary and discretionary factors and scale in data envelopment analysis. European Journal of Operational Research, v. 158, p 20-33, 2004.

Artigo Recebido em 10/08/2004 e aceito para publicação em 17/08/2005. 